\title{
The reciprocal value of Doctoral Design Research when housed within a Creative Business Center: a case study in Porto, Portugal.
}

\author{
Alvelos, Heitor ${ }^{a}$; Barreto, Susana ${ }^{b}$ and São Simão, Fátima ${ }^{c}$ \\ ${ }^{a}$ Department of Design, Universidade do Porto, Portugal, ${ }^{b}$ Department of Design, \\ Universidade do Porto, Portugal, ${ }^{\mathrm{c}}$ Science and Technology Park, Universidade do Porto, \\ Portugal.
}

\begin{abstract}
This paper presents the case study of a pilot partnership between a Doctoral Program in Design and a Science and Technology Park located in Porto, Portugal. The partnership has been in effect since 2013; the Doctoral Program has resided within the premises of the Science and Technology Park, as an immersive research environment, in order to foster knowledge transfer and collaborations through gradual, mutual and informal recognition of reciprocal assets.
\end{abstract}

We contextualise the premises and stakeholders in this partnership, outline the empirical methodologies employed, and provide examples of selected projects that illustrate the implementation of these methodologies.

Furthermore, we provide an inventory of challenges detected throughout the development of the partnership, as well as information on how these challenges are being overcome.

We conclude by summarising the main accomplishments of this partnership, as well as outlining validation by various external agents and opening up prospects for future development. These will include thorough materials that may be of use for implementation in other academic, scientific and entrepreneurial environments.

Keywords: Design research; Doctoral research; entrepreneurship; knowledge transfer; creative industries. 


\section{Introduction}

This paper presents the case study of the Doctoral Program in Design [PhDDesign] of the University of Porto [UP] as mainstay of an experiment in knowledge transfer. Based at UP's Science and Technology Park [UPTEC] since 2013, PhDDesign has co-developed the hypothesis that for Design Research to flourish and consolidate itself as an Applied Science Field, it may benefit from a primary strategic location within a creative industries center.

A research question may therefore be summarised as follows:

Can the bridges between design research and industry be most effectively consolidated by primarily hosting $\mathrm{PhD}$ research in an entrepreneurial environment, rather than an academic one?

The objectives of the experiment can be summarised as follows: - To contribute to the consolidation of a culture of applied design research; - To contribute to a rooting of reciprocal collaborations and added value between design research and contemporary entrepreneurial environments; - To contribute to the legitimisation and credibility of design research as a multidisciplinary interface.

\section{Context}

PhDDesign was created and formally accredited in 2011 at the University of Porto. The course is biennial with three editions as of January 2017. Its premise is the consolidation of a recent culture of design research, in particular with regards to the potential for regional scale development (in partnership with the University of Aveiro and ID+, Research Institute in Design, Media and Culture), as well as its binding potential with the entrepreneurship sphere - in particular in the emerging ecosystem of micro, small and medium-sized enterprises [SMEs] in creative fields.

UPTEC is a structure for supporting knowledge transfer between University and market. It fosters the creation of technological, scientific and creativity based companies, enabling multiple relationships between Portuguese and international innovation centres. UPTEC has been providing a vital contribution to the social and economic leverage of knowledge produced at the University of Porto, and has already supported over 450 business ideas, hosting about 200 projects every year. In 2013, UPTEC won the RegioStars Award, which identifies good practices in regional development and highlights original and innovative projects that may be inspiring to other regions. Through its engagement with creative businesses, the key role of design as business opportunity and a fundamental tool for 
market differentiation to any company (regardless of its economic sector or stage of development) has gradually become evident in the context of UPTEC.

The above frameworks led to the submission of PhDDesign to the Foundation for Science and Technology [FCT] call "Doctoral Programmes" in February 2013. UPTEC was presented as the interface partner for the development of an exploratory culture of collaboration; a strategic emphasis was placed on its Creative Center [UPTEC PINC].

The application earned an excellent evaluation, with a 9/10 rating on all four evaluation parameters. Comments by the international evaluation jury included the following statement:

"The results of the program could have an important positive social and industrial impact on the Portuguese and international scene". FCT (2013).

Following this application, the Vice-Rector for Research and Development of U.Porto supported the physical placement of the PhDDesign at UPTEC PINC, as a way of fostering the promise contained in the application. This residency was implemented in September 2013, with the full support of the Executive Directorate of UPTEC and the UPTEC PINC infrastructure. Since then, 17 students have been conducting their research in an immersive environment, while enhancing its intersection with the functional and prospective universe of UPTEC. The fact that 14 of these students' research has been fully funded by FCT ensures the desired level of commitment.

\section{Methodologies}

The approach to the challenge has been threefold:

- Ensuring the admission and development of doctoral projects best suited to knowledge transfer and using design research as a multidisciplinary interface; a research proposal is required upon application, and candidates are invited to clarify their proposals' potential for implementation and development. Subsequent project development of accepted students follows up on this ambition.

- Ensuring curricular content and activity that consolidates the stated reciprocal mission; this has included the mapping of spatial dynamics at UPTEC centers, design and consultancy services with companies and the UPTEC School of Startups, a series of printed materials focusing on good practice in communication environments, and the joint envisioning of innovative strategies of institutional communication.

- Fostering extra-curricular contexts of conviviality and opportunities for collaboration; these have included the creation of a small permaculture farm (subsequently maintained by 
startup company Noocity), holding a series of free-access, hands-on workshops (FuturePlaces medialabs), and the co-authorship of projects (ethno-branding project Porto Pelo Porto with contributions from FAHR021.3, The FuturePlaces Fountain Project in coordination with Still Urban Design, and Radio Manobras, an independent, community-led radio station that is both a $\mathrm{PhD}$ case study and the prospective host of a set of radio interviews on the subject of design research).

The above fronts are articulated and monitored on a primarily empirical basis, with regular scientific board meetings where a calibration is performed as required by specific instances. It is also worthy of note that the above approaches are reciprocally contributive between themselves; as an example, the farm began as an informal context, and has meanwhile been scientifically validated as a case study by one of the doctoral researchers.

\section{Case Studies (sample)}

We summarise three sample cases that illustrate the above methodological geometry into a tangible framework, while revealing examples of mechanics and challenges that emerge empirically.

- The re-capacitation of regional traditional industry through implementation of startup principles and customised design processes (an example of fostering the recognition of collaborative opportunities)

This pilot project currently involves UPTEC and PhDDesign as strategic partners for the recovery of a traditional shoe factory in the greater Oporto area - that, like many others, faces imminent bankruptcy. This study is meant to be a testbed for the possibility of implementing selected startup principles as a mechanism of modernisation and flourishment of an ailing traditional industry sector. Complementary input is provided by PhDDesign students Abhishek Chatterjee and António João Gomes within the scope of their theses, and post-doctoral researcher Pedro Carvalho de Almeida, whose research focuses on inventories and narratives towards the recognition of local brands as cultural heritage and economic assets.

- Ascertainment of the potential impact of design on [medical pathology designation withdrawn for issues of confidentiality] (an example of doctoral projects best suited to knowledge transfer and design research as a multidisciplinary endeavour)

A doctoral research project, admitted to the doctoral program in 2015, concerns design for a specific medical condition. Within the Course strategy, the project signals a multidisciplinary approach, as it is receiving co-supervision in medical fronts, and consolidates an emerging interest and potential for patenting. 
- UPTECX+ (an example of curricular activity that consolidates the reciprocal mission)

As part of the celebration of ten years of UPTEC (slated for September 2017), PhDDesign students were invited to envision unexpected ways of communicating UPTEC - with the possibility of future extrapolation towards broader institutional communication. The briefing emphasises the character of the expected outcomes: open-ended, experimental, bold - while remaining conceptually sound. Three research challenges were posed to the students:

1. What, in my UPTEC experience, needs input / improvement / change on a design level?

2. What competences do I have that may be of use to communicate an institutional environment in 2017 ?

3. How can my research be connected to this challenge? Can this become a case study of my own?

Following a series of field work sessions with companies (including informal interviews and data collection), students are currently developing a glossary of new terms for entrepreneurship, an ongoing installation with contributions from incubated companies (present and future), and a promotional video that strengthens semantic ties with local culture. Furthermore, the outputs will inform the development of UPTEC's institutional rebranding.

\section{Main Challenges}

The empirical work developed since late 2013 has revealed a set of challenges: while expected as a whole, their specificity can only be managed as live processes of interpretation and incorporation.

A gap is yet to be overcome between students' general availability and willingness to engage in exploratory fronts, and the companies' frequent unavailability due to professional commitments, deadlines and the imperative of profit. A clear path is the identification and unequivocal communication of reciprocal value, i.e., how can researchers and companies benefit by collaborating in exploratory environments? Sensitive intermediation is necessary from both ends (namely by the PhDDesign and UPTEC coordinators), and simple clear rules have to be in place to make sure that researchers and companies fully understand the goals and limits of such collaboration in order to avoid potential conflicts and liberties: neither are the researchers to be regarded as permanently available design consultants, nor should the companies' availability and data be regarded as readily accessible (various of these projects have very few resources, human and otherwise, as well as confidential information that cannot be shared); copyright issues should also be closely monitored. 
These challenges are being addressed on an individual basis, as each project and prospective collaborations raise distinct issues.

The time span of the originally proposed objectives has revealed a certain degree of overambition: the implementation of a fully flourished design research culture, as well as its concurrent recognition of affinities and complementarities with industry, has required a temporal adjustment due to the intrinsic complexity of the various stakeholders and the relative absence of prior templates. A pedagogy of applied design research in entrepreneurial environments needs to be regarded as a longer-term challenge. The same applies to the development of an entrepreneurial culture that regards the coexistence with design research as a competitive advantage for its businesses.

A more balanced approach will be required when it comes to geographical and semantic relocation of the course; despite the clear advantages and strategic advancements, the maintenance of PhDDesign in academic environments should not be bypassed entirely. This is being addressed through the organisation of exhibitions, the hybridisation of the PhDDesign student body with students from other PhD programs (and Masters courses), and the organisation a series of annual conferences on design research ("UD”).

\section{Conclusions and future prospects}

This pilot partnership in knowledge transfer has already proven to be informative in external contexts: namely, Universities and courses that have come in contact with it, through project co-supervision, external examination, guest lecturing, mobility programs, and forum attendance. Various aspects of this partnership are fostering cooperation of various scales and natures with other academic environments, through validation, replication, and joint strategic enquiry. There various typologies of cooperation will be the subject of further communication.

At the time of writing, we are able to signal cooperation with the following academic fronts:

- $\quad$ the University of Aveiro, Portugal (partner institution in various curricular units, joint conference organisations, and scientific consultancy);

- $\quad$ the University of Coimbra, Portugal (namely in the reciprocal collaboration with the Doctoral Program in Human Rights in Contemporary Societies, expressed in cosupervisions and keynote addresses);

- $\quad$ the University of Lisbon (forum keynotes and think-tanks for implementation of aspects of PhDDesign as course templates elsewhere);

- $\quad$ the University of Brighton (reciprocal consultancy with Faculty of the College of Arts and Humanities); 
- $\quad$ Central Saint Martins / University of the Arts London (prospective co-supervisions and consultancy towards the consolidation of knowledge transfer at $\mathrm{PhD}$ research level); - $\quad$ the University of Saint Joseph, Macau (by integrating its Dean of Creative Industries on the Course Advisory Board, as well as welcoming staff and students into research workshops co-organised with the Macau Institute of European Studies); - $\quad$ the inclusion of Professor Jon Wozencroft (Royal College of Art, London) in the Course Advisory Board;

- $\quad$ Doctoral co-supervision by a Professor of Product Design from Istanbul Technical University;

- $\quad$ Aalto University: prospective cooperation with the PhD Program hosted by Aalto University School of Arts, Design and Architecture / EMPIRICA Research Group;

- $\quad$ UTAustin|Portugal: student involvement in numerous workshops conducted within the framework of futureplaces medialab (a partnership the University of Texas at Austin).

We overall regard the partnership between PhDDesign and UPTEC as successful in the accomplishment of its original premises; its pioneering nature, as well as the various projects and fronts that have been bearing fruit, have been further validated by both institutions, collaborating partners and associated agents.

However, the project will require further development in order to fully accomplish its goals, namely by following up on course graduates' own paths of knowledge transfer and consolidation of value. Similarly, it seems premature to fully assess the mutual benefit of the interchange between companies and $\mathrm{PhD}$ research. A number of relationships between both entities have been forged that point towards longer-term spans and outcomes. Nevertheless, impact may be regarded as immediate on specific fronts: examples are the demand for design consultancy by incubated companies, and the individual networks that students have been developing with startups that may be of relevance to their research.

Given the exploratory nature of this partnership, and in the absence of a prior template, challenges and the need for adjustments are inventoried and acted upon by the Course Scientific Board in coordination with the UPTEC executive directors.

A thorough report is currently under production, in cooperation with the course's Advisory Board, with completion set for late 2017, after the graduation of the first set of students admitted in 2013. Besides a balance of activities and outcomes, we intend to include a set of recommendations for implementation by interested parties within analogous contexts.

This pilot partnership in knowledge transfer has already proven to be informative in external contexts: namely, Universities and courses that have come in contact with it, through project co-supervision, external examination, guest lecturing, mobility programs, and forum attendance. 
The authors wish to thank the Foundation for Science and Technology for the financial support of PhDDesign through the grant ref. PD/00048/2012.

\section{References}

Alvelos, H. (n.d.). PhDDesign. Retrieved from http://endlessend.up.pt/phddesign

Alvelos, H., Carvalho de Almeida, P., et al (2015). Porto Pelo Porto. Retrieved from http://portopeloporto.org

Alvelos, H., \& Melo, R. (2015) UD15: Periphery and Promise. Retrieved from http://endlessend.up.pt/ud15

Alvelos, H., \& São Simão, F. (n.d.). FuturePlaces - medialab for citizenship. Retrieved from http://futureplaces.org

Cortesão, J. \& Fernandes, S. (n.d.). Still Urban Design. Retrieved from http://www.stillurbandesign.com

Directorate-General for Education and Culture, European Commission, and Hogeschool vor de Kunsten Utrecht (2010). The Entrepreneurial Dimension of the Cultural and Creative Industries. Retrieved from http://bit.ly/2panUgw

European Commission (2013). REGIO STARS 2013 winner - Smart Growth: Science and Technology Park of the University of Porto (UPTEC) - North of Portugal (Norte), Portugal. Retrieved from http://bit.ly/2pafLsi

Foundation for Science and Technology (n.d.). FCT PhD Programmes. Retrieved from https://www.fct.pt/apoios/programasdoutoramento/index.phtml.en

Foundation for Science and Technology (2013). Evaluation for PD/00048/2012. Academic funding report.

Frois, F., \& Reis, H. (n.d.). FAHR 021.3. Retrieved from http://www.fahr0213.com

KEA European Affairs (2006). The Economy of Culture in Europe. Retrieved from http://www.keanet.eu/ecoculture/executive_summary_en.pdf

Ruivo, J. (n.d.). Noocity. Retrieved from http://www.noocity.com

Radio Manobras (n.d.). Radio Manobras. Retrieved from http://radiomanobras.pt

University of Porto (n.d.). Science and Technology Park of University of Porto. Retrieved from http://uptec.up.pt 